Journal of Fundamental and Applied Sciences

ISSN 1112-9867

Available online at

http://www.jfas.info

\title{
ECO-CASTING OF AEOLIAN BLADES AND SOLAR PANELS WITH COMPOSITES MATERIALS VIA RTM TECHNOLOGY
}

\author{
B. Attaf \\ Expert en Structures Composites, Région PACA - France
}

Received: 01 February 2010 / Accepted: 02 June 2010 / Published online: 30 June 2010

\begin{abstract}
The technique used for manufacturing composite wind turbine blades and solar panels must be sure of environment-friendly. In order to achieve this objective, the closed mould manufacturing process that takes into account environment preservation and health protection besides assurance quality will be the subject of this article. The requirements of sustainable development and ecodesign are the objectives to be fulfilled with an acceptable degree of tolerance in relation to the new regulations and ecostandards.
\end{abstract}

Keywords: composite materials; ecodesign; RTM process; permeability; sustainable development; renewable energy

\section{INTRODUCTION}

Dans l'industrie moderne de fabrication des pales d'éoliennes et panneaux solaires, les matériaux composites à base de fibres occupent une place prépondérante, car ils présentent des avantages attractifs résidant principalement dans les bonnes caractéristiques mécaniques, thermiques et hygrométriques associées aux exigences de légèreté, de coût et de meilleure durée de vie. En plus de ces critères techniquement qualitatifs, s'ajoutent les exigences d'une méthode de fabrication sûre et respectueuse de l'environnement et de la santé.

Author Correspondence, e-mail: b.attaf@wanadoo.fr

ICID: 1037254 
C'est dans ce contexte que les procédés à moule fermé permettent de répondre aux exigences de développement durable et d'éco-conception, car les avantages offerts par ces procédés modernes (moule fermé) par rapport aux procédés artisanaux (moule ouvert) résident, d'une part, dans l'accroissement de la productivité et d'autre part, dans la réduction des quantités d'émission de composés organiques volatils (COV). Par ailleurs, le respect de l'environnement favorise considérablement le développement de ces procédés qui ouvrent de grandes perspectives industrielles et technologiques dans les domaines éolien et solaire. Parmi ces procédés de fabrication, nous citerons le procédé RTM (Resin Transfer Moulding = moulage par transfert de résine) sur lequel nous focaliserons notre attention.

Ce procédé qui consiste à injecter de la résine sous forme liquide dans une empreinte fermée (moule/contre moule), dans laquelle le renfort fibreux sec (préforme) a été préalablement placé [1], s'appuie principalement sur l'utilisation de la différence de pression (ou perte de charge) qui se manifeste à l'intérieur de l'empreinte fermée, permettant ainsi l'écoulement de la résine et par conséquent l'imprégnation du renfort fibreux [2]. La Figure 1 montre les principales étapes du procédé RTM que nous avons résumé par :

- Etape 0 : sélection du renfort fibreux recommandé par le bureau d'études

- Etape 1: préparation du préforme (orientation des fibres et séquence d'empilement)

- Etape 2 : fermeture du moule après placement du renfort et mise à vide

- Etape 3 : injection de la résine et imprégnation progressive du renfort jusqu'au remplissage

- Etape 4 : polymérisation, séchage et durcissement de la résine (cuisson)

- Etape 5 : ouverture du moule et démoulage de la pièce

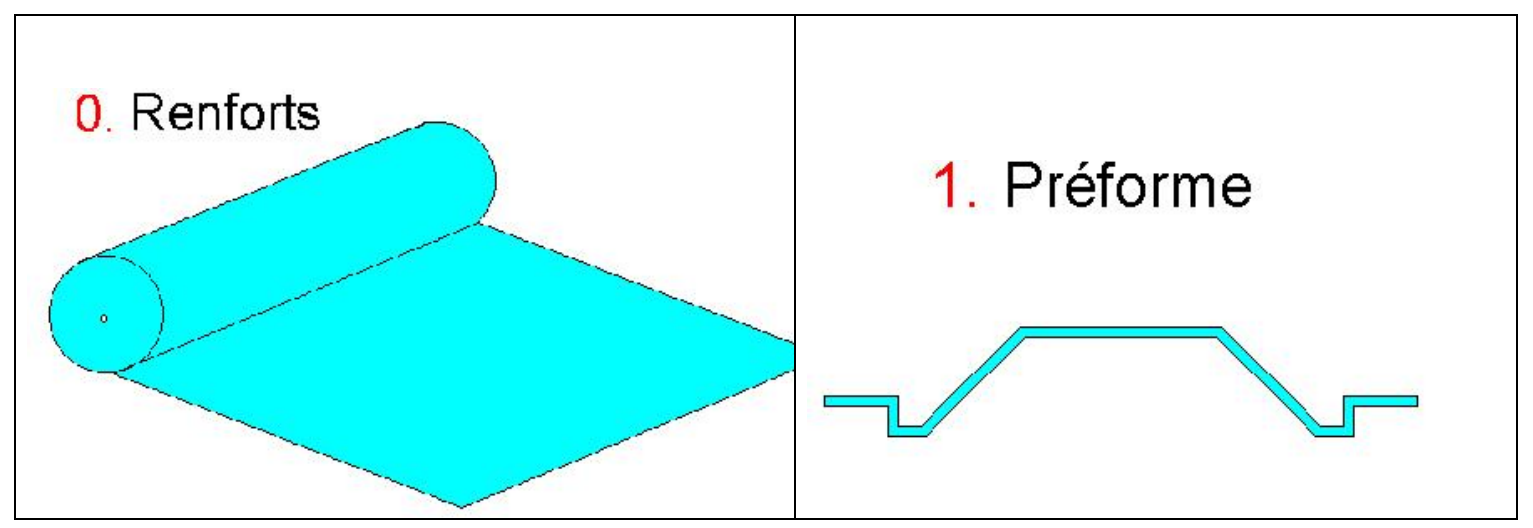




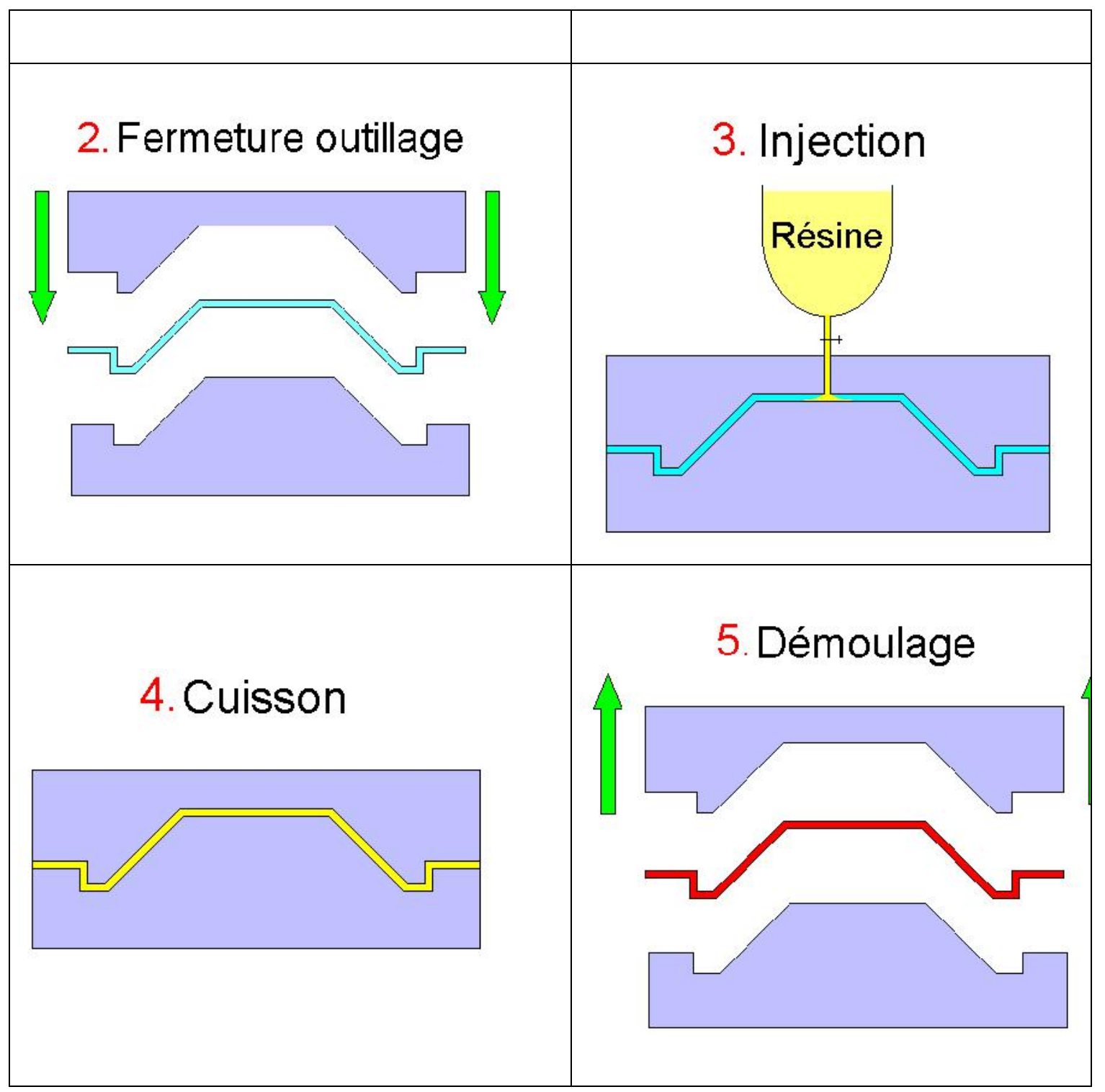

Fig.1. Différentes étapes de réalisation d'une pièce composite par le procédé RTM

L'étape 3 s'avère être une étape importante dans le processus de réalisation d'une pièce en matériaux composites. Cette étape d'injection et d'écoulement de la résine dans le milieu fibreux s'appuie sur l'utilisation de la loi de Darcy qui se caractérise par la valeur de perméabilité de la résine, $K$, qui est une caractéristique physique représentant la capacité de transfert de fluide à travers le matériau considéré. Cette perméabilité dépend de plusieurs paramètres : la nature du renfort, le sens et la disposition des fibres, la séquence d'empilement des plis, la température de la résine, le positionnement des points d'injection-aspiration, etc. Cependant, la simulation du comportement de l'écoulement dans un milieu fibreux anisotrope doit être étudiée avec précaution, car les valeurs de perméabilité sont des valeurs à définir avec précision. Une erreur mineure 
dans l'évaluation de ces valeurs peut engendrer des écarts considérables qui ne peuvent être acceptés dans la pratique.

\section{Formulation de la perméabilité (loi de darcy)}

\section{Principe de mesure de la perméabilité}

En 1856, Henry Darcy a démontré que pour un fluide Newtonien incompressible en écoulement laminaire, la vitesse à laquelle ce fluide se conduit dans un milieu homogène isotrope est proportionnelle au gradient de pression $\Delta P / \Delta L$ et inversement proportionnelle à sa viscosité dynamique $\mu$ (Figure 2).

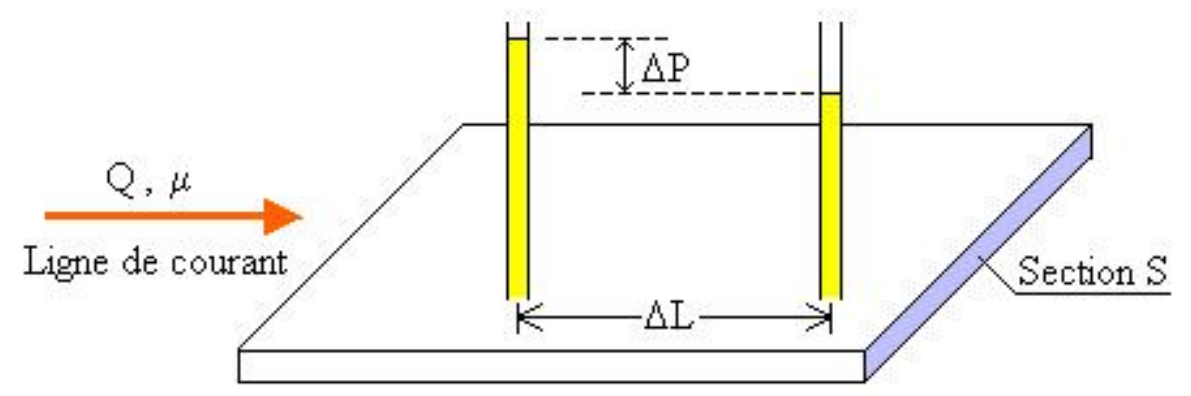

Fig.2. Mesure de la perméabilité

La vitesse de conduite du fluide pour un débit $Q$ s'écoulant à travers une section $S$ est exprimée par:

$$
v=\frac{Q}{S}=\frac{K}{\mu} \times \frac{\Delta P}{\Delta L}
$$

où,

$v$ : vitesse du fluide $(\mathrm{m} / \mathrm{s})$

$Q:$ débit $\left(\mathrm{m}^{3} / \mathrm{s}\right)$

$S:$ section de l'écoulement $\left(\mathrm{m}^{2}\right)$

$K$ : perméabilité $\left(\mathrm{m}^{2}\right)$

$\mu$ : viscosité du fluide (Pa.s)

$\Delta P / \Delta L:$ perte de charge $(\mathrm{Pa} / \mathrm{m})$

L'expérience de Darcy est caractérisée par la variation de débit $Q$ en fonction de la perte de charge $\Delta P$. Sa représentation graphique est illustrée par la Figure 3. 


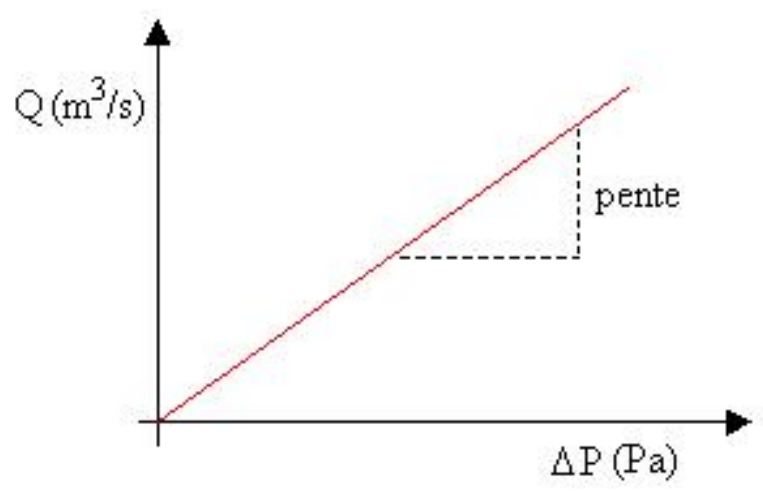

Fig.3. Courbe débit-pression

La perméabilité est définie expérimentalement par :

$$
K=\frac{\mu \times \Delta L}{S} \times(\text { pente })
$$

En remplaçant la pente par sa valeur $Q / \Delta P$, on aura :

$$
K=\frac{\mu \times \Delta L}{S} \times \frac{Q}{\Delta P}
$$

Si on prend, à titre d'exemple, les valeurs suivantes :

$\Delta L=0.2 \mathrm{~m} \quad S=0.001 \mathrm{~m}^{2} \quad \quad \mu=50 \times 10^{6} \mathrm{~Pa} . \mathrm{s} \Delta P=0.2 \times 10^{6} \mathrm{~Pa} \quad Q=2 \times 10^{-6} \mathrm{~m}^{3} / \mathrm{s}$ et en appliquant la relation (3), on trouve une valeur de perméabilité $K=\mathbf{1 0 0} \times \mathbf{1 0}^{-12} \mathbf{m}^{2}$

\section{Perméabilités longitudinales et transversales (Figure 4)}

La loi de Darcy généralisée s'écrit sous la forme compacte suivante [3, 4] :

$$
\bar{v}=-\frac{1}{\mu} K \nabla P
$$

ou sous la forme développée suivante :

$$
\left\{\begin{array}{l}
\mathrm{v}_{x} \\
\mathrm{v}_{y}
\end{array}\right\}=-\frac{1}{\mu}\left[\begin{array}{ll}
K_{x x} & K_{x y} \\
K_{x y} & K_{y y}
\end{array}\right]\left\{\begin{array}{l}
\partial P / \partial x \\
\partial P / \partial y
\end{array}\right\}
$$

où le tenseur de perméabilité $K$ dépend de l'angle d'orientation des fibres $(\theta)$ 
- dans le repère d'orthotropie $(\theta=0)$ :

$$
\left[\begin{array}{ll}
K_{x x} & K_{x y} \\
K_{x y} & K_{y y}
\end{array}\right]=\left[\begin{array}{cc}
K_{11} & 0 \\
0 & K_{22}
\end{array}\right]
$$

- dans un repère quelconque $(\theta \neq 0)$ :

$$
\left[\begin{array}{ll}
K_{x x} & K_{x y} \\
K_{x y} & K_{y y}
\end{array}\right]=\left[\begin{array}{ll}
K_{11} C^{2}+K_{22} S^{2} & \left(-K_{11}+K_{22}\right) C S \\
\left(-K_{11}+K_{22}\right) C S & K_{11} S^{2}+K_{22} C^{2}
\end{array}\right]
$$

avec $: \mathrm{C}=\cos \theta, \mathrm{S}=\sin \theta$ et $K_{i j}=\frac{1}{k_{i j}} \frac{R_{f}^{2}}{4} \frac{\left(1-V_{f}\right)^{3}}{V_{f}^{2}} \quad(i, j=1,2)$

où : $k_{i j}, R_{f}$ et $V_{f}$ correspondent respectivement à la constante de Kozeny, au rayon de fibre et à la fraction volumique des fibres.

La perméabilité moyenne transversale (à travers l'épaisseur d'un préforme composé de plusieurs plis $K_{i j}^{1}, K_{i j}^{2}, K_{i j}^{3}, . . K_{i j}^{n}$ ) peut être calculée suivant la règle des moyennes :

$$
\bar{K}_{i j}=\frac{1}{H} \sum_{k=1}^{n} h^{k} K_{i j}^{k}
$$

où $H$ et $h^{k}$ correspondent respectivement à l'épaisseur totale du préforme et à l'épaisseur du pli $k$.

Dans la plupart des cas, les pièces composites ont une épaisseur mince par rapport à leurs autres dimensions (longueur et largeur). Par conséquent, la variation de débit suivant l'épaisseur peut être négligée.

Une fois les perméabilités connues, une combinaison de l'équation de la loi de Darcy avec l'équation de continuité, permet de développer l'équation gouvernant la distribution de la pression. Cette équation s'écrit sous la forme compacte suivante :

$$
\nabla \cdot\left(\frac{K}{\mu} \nabla P\right)=0
$$

Sous la forme développée, cette équation s'écrit : 


$$
\frac{\partial}{\partial x}\left(\frac{K_{x x} \partial P}{\mu \partial x}\right)+\frac{\partial}{\partial x}\left(\frac{K_{x y} \partial P}{\mu \partial y}\right)+\frac{\partial}{\partial y}\left(\frac{K_{y x} \partial P}{\mu \partial x}\right)+\frac{\partial}{\partial y}\left(\frac{K_{y y} \partial P}{\mu \partial y}\right)=0
$$

Le système d'équation non-linéaire obtenu, peut être résolu par la méthode itérative. Les conditions aux limites représentatives du système, sont [2] :

- au point d'injection : $\quad P=P_{o}$

- au front de débit : $\quad P=0$

- à l'extrémité du moule : $\quad v=-\frac{1}{\mu}\left(K_{n n} \frac{\partial P}{\partial n}+K_{n t} \frac{\partial P}{\partial t}\right)=0$

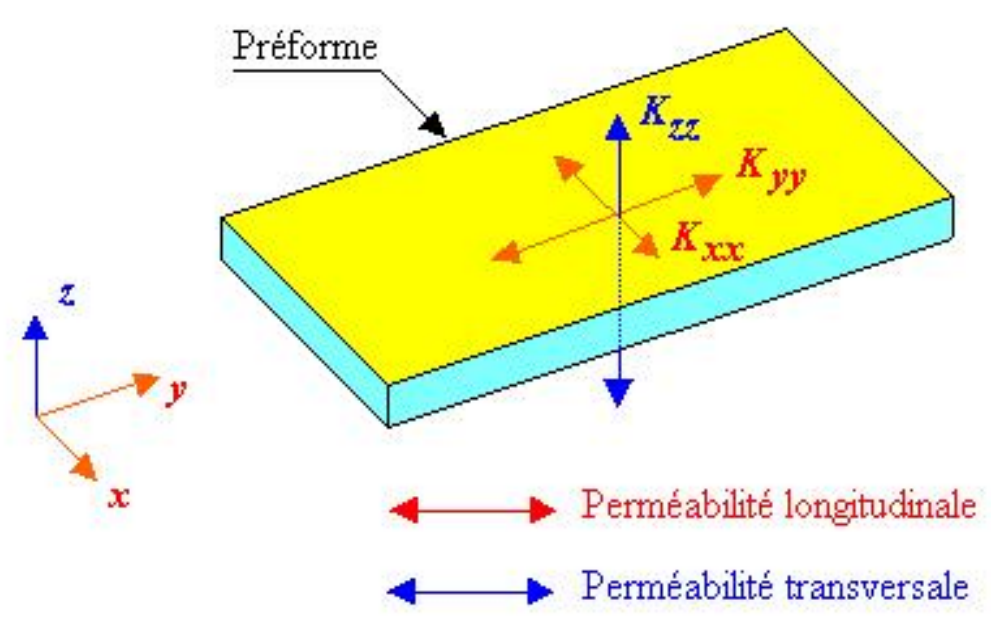

Fig.4. Perméabilités longitudinales et transversales

\section{RESULTATS ET DISCUSSION}

Afin de donner une idée sur le comportement de l'écoulement latéral de la résine durant le stage de remplissage d'un renfort fibreux anisotrope, une plaque carrée constituée de un ou deux plis orientés différemment a été considérée.

La Figure 5 représente le positionnement des points d'injection et d'aspiration par rapport au centre de la plaque. L'injection est centrale pendant que l'aspiration s'effectuée au niveau des quatre coins de la plaque. Le choix des points d'aspiration sur les quatre coins a été établi dans le but d'équilibrer la répartition uniforme du flux d'aspiration. Par conséquent, les résultats obtenus ne seront pas affectés par le choix de positionnement des points d'injection-aspiration. 


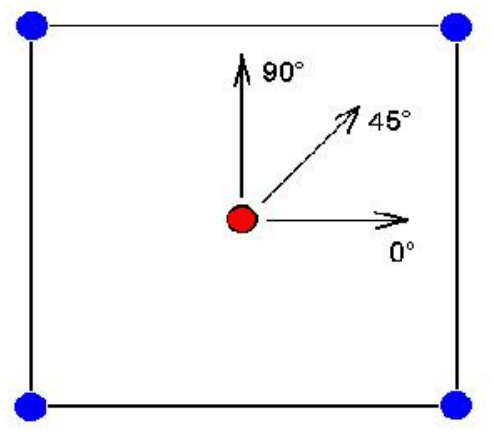

: Point d'aspiration

: Point d'injection

Fig.5. Injection centrale et aspiration sur les quatre coins de la plaque

Le comportement de la résine en mouvement est simulé grâce à la relation 6 . La représentation graphique du champ de pression résultant est donnée sous forme de couleurs différentes.

\section{Comportement du champ de pression : cas d'un pli unidirectionnel}

D'après la Figure 6 et pour les trois cas examinés $\left(\theta=0^{\circ}, \theta=90^{\circ}\right.$ et $\left.\theta=45^{\circ}\right)$, on remarquera que l'écoulement de la résine suivant la direction des fibres $\theta=0^{\circ}$ est plus important que les autres directions car c'est le taux volumique des pores suivant le sens longitudinale des fibres qui définit les cheminements préférentiels de l'écoulement de la résine au sein du milieu poreux. Cet écoulement sera faible pour le cas où $\theta=90^{\circ}$ car les fibres créent un obstacle pour l'avancement de la résine. Cet obstacle est caractérisé par un faible taux de présence des pores dans cette direction, favorisant un maintien mais non une augmentation de la conductivité de la résine. Cependant, l'idée d'un écoulement de la résine d'une façon concentrique par rapport au point d'injection n'est représentative pour un renfort fibreux unidirectionnel orthotrope. 


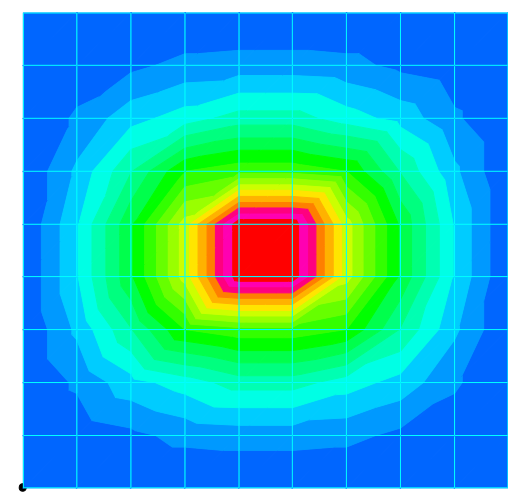

Pli orienté à $\theta=0^{\circ}$

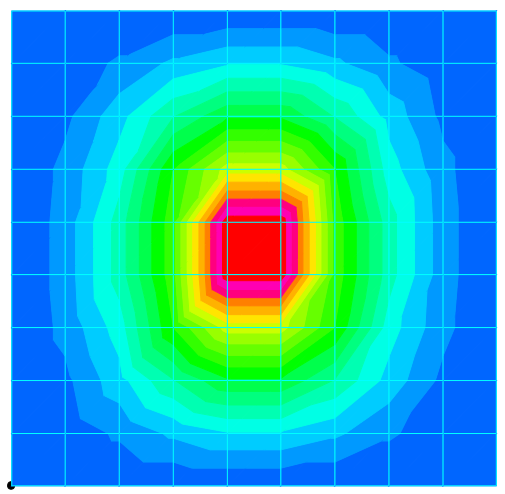

Pli orienté à $\theta=90^{\circ}$

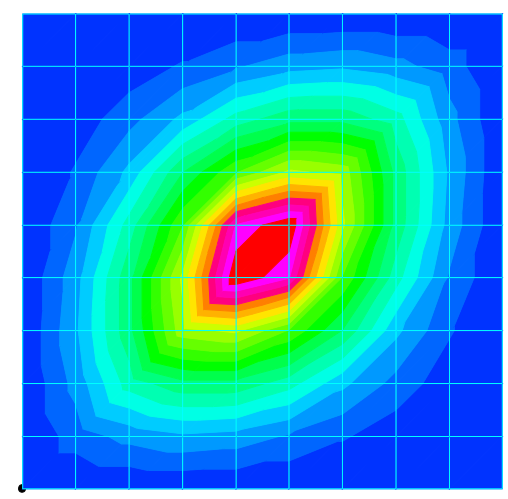

Pli orienté à $\theta=45^{\circ}$

Fig.6. Cas d'un pli unidirectionnel avec différentes orientations

\section{Comportement du champ de pression : cas d'un stratifié à renfort unidirectionnel}

Pour cet exemple, le positionnement des points d'injection-aspiration reste identique à celui représenté par la Figure 5. Mais le nombre de plis constituant le stratifié est fixé cette fois-ci à deux ; où chaque pli est orienté d'un angle différent.

Quatre cas avec alternance de plis ont été examinés : $\left[45^{\circ} / 90^{\circ}\right],\left[45^{\circ} / 0^{\circ}\right]$, $\left[90^{\circ} / 0^{\circ}\right]$ et $[-$ $45^{\circ} / 45^{\circ}$. Les résultats sont présentés sur la Figure 7. Cette dernière montre que l'écoulement résultant est dominant pour le cas d'empilement offrant une meilleure distribution des pores dans la structure du renfort, ce qui engendre par conséquent une meilleure conductivité de la résine. Le drainage de la résine est anisotrope, et plus rapide et plus important dans le sens des fibres. En outre de la direction des fibres, il faut noter que la perméabilité dépend aussi de l'ordre d'empilement des plis (perméabilité transversale). 


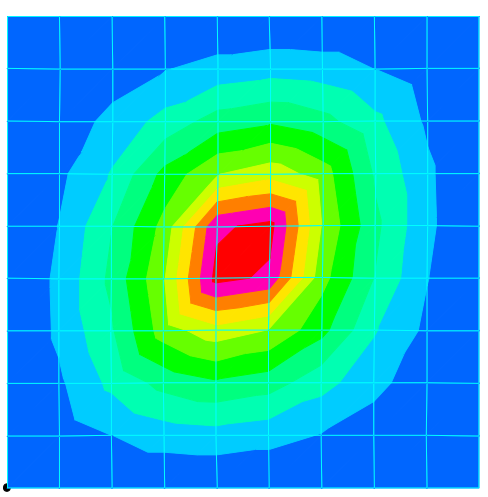

Stratifié : $\left[45^{\circ} / 90^{\circ}\right]$

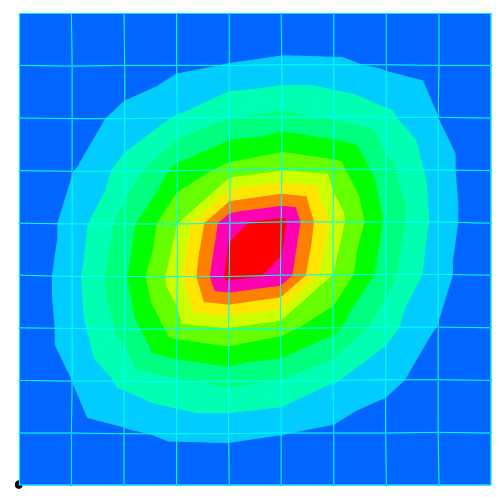

Stratifié : $\left[45^{\circ} / 0^{\circ}\right]$

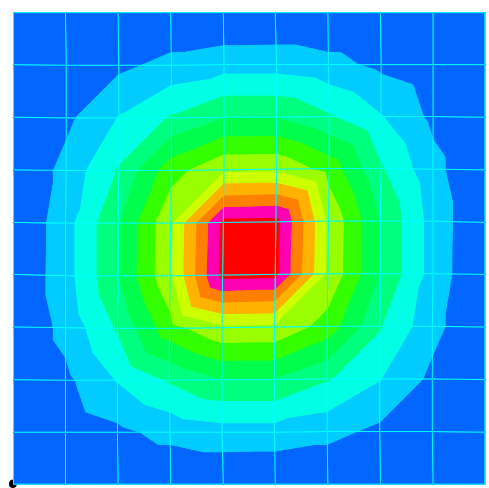

Stratifié : $\left[-45^{\circ} / 45^{\circ}\right]$

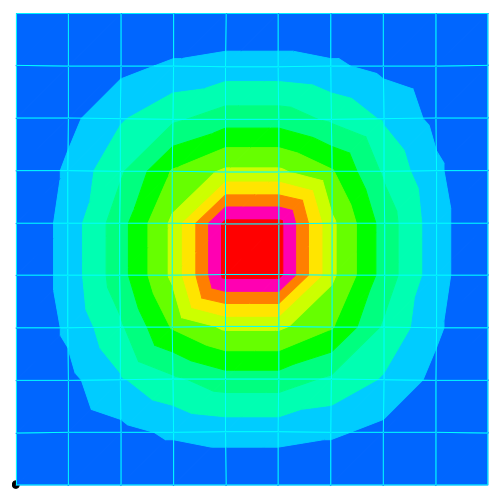

Stratifié : $\left[90^{\circ} / 0^{\circ}\right]$

Fig.7. Cas d'un stratifié composé de deux plis unidirectionnels orientés à des angles différents

Le choix d'un empilement adéquat est défini habituellement par un calcul de structure par éléments finis et les résultats de calcul doivent répondre aux critères exigés par le cahier des charges. Cependant, toute modification de l'alternance des plis peut, d'une part, favoriser le processus de drainage de la résine mais peut, d'autre part, affecter les caractéristiques mécaniques du matériau et par conséquent la résistance du produit obtenu. Le choix final de la séquence d'empilement doit répondre simultanément et favorablement aux conditions retenues par les calculs et celles imposées par les principes du procédé RTM. Cette question particulière doit faire l'objet d'une étude impérative avant la mise en œuvre du procédé. 
L'implantation des valeurs exactes de perméabilité dans les logiciels de calcul de simulation de l'injection, permet de donner une représentation plus conforme à la réalité du comportement de l'écoulement. Les courbes représentatives du champ de pression peuvent être visualisées pli par pli ce qui n'est pas le cas pour certains logiciels commerciaux de simulation. Cependant, les futurs logiciels de simulation du transfert de la résine doivent tenir compte de ces suggestions afin de donner une approche correcte et conforme au cas réel. D'autre part, la forme géométrique de la structure, la variation de la température et le positionnement des points d'injection-aspiration sont d'autres paramètres qui peuvent aussi influencer le phénomène du transfert de la résine et le comportement de l'écoulement. Ces paramètres doivent faire l'objet d'études de recherche et développement.

\section{CONCLUSION}

Le procédé RTM présente une solution industrielle à la production des pales d'éoliennes et panneaux solaires en matériaux composites avec un coût réduit et une cadence de production modeste. C'est un procédé propre et non polluant permettant un gain de temps considérable par rapport aux techniques traditionnelles. Les pièces obtenues (monoblocs) ont une excellente qualité d'aspect des deux faces et de meilleures caractéristiques mécaniques. Ces avantages ont attiré l'attention de plusieurs industriels et ont ouvert de grandes perspectives pour la fabrication des pales d'éoliennes et panneaux solaires de grandes dimensions.

Afin de respecter les nouvelles réglementations mises en vigueur en terme du respect de l'environnement et de la protection de la santé, les entreprises s'engageant sur la voie de mise en œuvre d'un système d'analyse environnemental sont encouragées à définir et développer les points suivants :

- Développer des procédés de fabrication propres en réduisant les émissions.

- Développer de nouveaux matériaux respectant l'environnement et ayant de hautes performances à coûts maîtrisés.

- Créer des normes spécifiques à la qualification, la réparation et la gestion de la fin de vie des pales d'éoliennes et panneaux solaires.

- Etablir une activité bénéfique entre l'université, les centres de recherche et l'industrie. 
- Transférer la technologie par la formation et l'information aux futurs ingénieurs travaillant dans le domaine des énergies renouvelables.

L'assurance qualité, la protection de la santé et la préservation de l'environnement sont devenus aujourd'hui des aspects interdépendants et indissociables. Ces trois aspects constituent les premiers facteurs qui répondent au concept du développement durable. Dans ce contexte, les pales d'éoliennes et les panneaux solaires vont dans ce sens par la production d'une énergie propre et renouvelable ; par ailleurs leur fabrication doit être sûre et respectueuse de l'environnement.

\section{RÉFÉRENCES}

[1] Advani S G. 1994, Flow and rheology in polymer composites manufacturing, Composite Materials Series, Amsterdam, Elsevier.

[2] Background information and liquid molding, Tutorial on polymer Composites Molding, Université de Delaware (disponible sur Internet).

[3] Chung H P. et al. Composites Science and Technology. 2003, 63, 1015-1026.

[4] Kris H. et al. applied science and manufacturing. 2002, 33, 959-969. 


\section{ECO-MOULAGE DES PALES D'EOLIENNES ET PANNEAUX SOLAIRES EN MATERIAUX COMPOSITES VIA LA TECHNOLOGIE RTM}

\section{RESUME}

La technique de fabrication des pales d'éoliennes et panneaux solaires en matériaux composites doit être sûre et respectueuse de l'environnement. Afin d'atteindre cet objectif, le procédé de fabrication à moule fermé avec la prise en compte de la préservation de l'environnement et de la protection de la santé et ce, en parallèle avec l'assurance qualité fera l'objet de cet article. Les exigences du développement durable et l'éco-conception sont les objectifs à accomplir avec un degré de conformité admissible par rapport aux nouvelles dispositions réglementaires et éco-normes.

Mots clés : matériaux composites, éco-conception, procédé RTM, perméabilité, développement durable, énergie renouvelable.

\section{How to cite this article}

Attaf B. Eco-casting of aeolian blades_and solar panels with composites materials via RTM technology. J Fundam Appl Sci. 2010, 2(1), 35-47. 